\title{
Plantas frecuentemente utilizadas en zonas rurales de la Región Amazónica centro occidental de Ecuador
}

\section{Plants frequently used in rural areas of the Amazon Region, western central Ecuador}

Juan C. Clavijo

Consultor Independiente, Ecuador

Patricio Yánez M.

Universidad Internacional del Ecuador, Ecuador

Universidad Iberoamericana del Ecuador, Ecuador

Autor para correspondencia: apyanez@ hotmail.com

Fecha de recepción: 24 de Febrero de 2017 - Fecha de aceptación: 5 de Junio de 2017

Resumen: el presente estudio aborda aspectos relacionados con el tipo y frecuencia de uso del recurso vegetal en predios ubicados en el occidente de la región amazónica ecuatoriana (provincias de Napo, Pastaza y Morona Santiago). Los datos se generaron en ciento veinte y dos propiedades de dos grupos de finqueros: unos pertenecientes a la iniciativa Socio Bosque y otros no, el trabajo de campo se realizó en dos fases entre febrero a marzo de 2014 y entre febrero a marzo del 2015. En la información recogida, se incluyeron aspectos socioeconómicos y frecuencias de uso de plantas en seis categorías de uso (comestibles, combustibles, para madera, sociales y rituales, tóxicas y/o venenosas, medicinales). Entre otros resultados, se evidencia que, generalmente, los propietarios usan con mayor frecuencia las plantas introducidas más que las nativas; factores como la presencia de habitantes de fuera de la zona unida al desconocimiento del uso de la flora nativa influyen en que ésta termine siendo menos frecuentemente utilizada en la zona.

Palabras Clave: región amazónica de Ecuador; especies vegetales nativas; especies vegetales introducidas; uso de la flora

\begin{abstract}
The present study deals with aspects related to the type and frequency of use of the vegetal resource in properties located in the western Ecuadorian Amazon region (provinces of Napo, Pastaza and Morona Santiago). The data were generated in one hundred and twenty two properties of two groups of farmers: some belonging to the Socio Bosque program and others not, fieldwork was carried out in two phases between February and March 2014 and between February and March 2015. Part of the information collected included socioeconomic aspects and frequencies of use of plants in six categories of use (edible, fuel, wood, social and ritual, toxic and / or poisonous, medicinal). Among other results, we observed that, generally, the owners use the introduced plants more frequently than the native ones; factors such as the presence of inhabitants from outside the area coupled with lack of knowledge about the use of native flora influence in this fact.
\end{abstract}

Key words: amazon region of Ecuador; native plant species; introduced plant species; use of flora 


\section{Introducción}

Las plantas silvestres y las semicultivadas son fundamentales para la vida de una gran parte de la población mundial, ya que proporcionan materiales de construcción de bajo costo, combustibles, suplementos alimentarios, medicinas, herramientas y fuentes de ingreso. Pese a la importancia que tienen, no se ha entendido bien lo vulnerables que son a la recolección y otros procesos sociales (Cunningham, 2001; Yánez, 1999).

La flora del Ecuador ha sido desde siempre reconocida por ser rica en plantas útiles; evidencias de esto se tienen en las crónicas de los misioneros que acompañaban a los conquistadores en las que se hacía referencia al uso que daban los indígenas a las plantas que crecían en estos territorios (de la Torre et al., 2008).

El uso de la flora nativa es una fuente importante de diversos recursos para la gente. Sin embargo, el desconocimiento sobre esta flora, debido a la falta de acceso a información sobre la misma y a no tener capacitación o educación sobre su manejo, pudiera ocasionar que la gente no la cultive y no la use y eventualmente promueva su pérdida introduciendo especies ajenas que pueden afectar a la flora nativa y al ecosistema local (Uquillas, 1981; Yánez, 2012; Bonete et al., 2016).

El presente estudio buscó abordar algunos aspectos socio ambientales y la dinámica del uso de la flora en los cantones Puyo, Tena y Palora, áreas donde la mayoría de gente que posee fincas han optado por vivir en las ciudades y dejar gradualmente de lado la utilización de flora nativa y muchas veces se mantienen solo cultivos de especies introducidas cuyos productos se pueden comercializar relativamente rápido (Uquillas, 1981; Clavijo-Páez, 2016).

\section{Materiales y Métodos}

En la Región Amazónica de Ecuador existen dos grupos humanos relativamente numerosos: los indígenas y los colonos. Los indígenas provienen de los pueblos ancestrales de esta región e incluyen ocho nacionalidades, poseen en gran parte vastos territorios cubiertos con bosques húmedos tropicales. Los colonos comienzan a habitar los ecosistemas amazónicos llegando de otras partes del país, principalmente entre 1970 a 1980, cuando comenzó la explotación petrolera. El patrón de uso de este grupo humano ha sido la conversión del bosque nativo a cultivos, pastos y la extracción de madera (Palacios y Malessa, 2010).

La presente investigación fue desarrollada en las parroquias de Ahuano, Chontapunta, Pano, Misahuallí, Puerto Napo y Tálag (cantón Tena, provincia de Napo) (Figura 1); Mera, Teniente Hugo Ortiz, Shell, Puyo, Tarqui (cantón Puyo, provincia de Pastaza) y Palora (provincia de Morona Santiago) (Figura 2), a un rango altitudinal comprendido entre 400 a 900 msnm y en ambientes correspondientes a bosque húmedo tropical (sensu Yánez, 2014).

El área de estudio fue escogida debido a que cuenta con predios de mediana superficie, es de relativamente fácil accesibilidad y cuenta con la presencia de pobladores permanentes (Granda, 2015; Clavijo, 2016; Yánez \& Granda, 2016). 
Entre febrero a marzo de 2014 y febrero a marzo de 2015 se efectuó el trabajo de campo en 122 predios elegidos al azar (centro-occidente de las provincias de Pastaza, Morona Santiago y Napo). Para la primera y segunda fase se aplicaron dos encuestas semiabiertas (Clavijo, 2016), mismas que permitieron registrar información socioambiental (en 122 fincas) y de uso cotidiano de la flora (en 66 fincas de las 122).

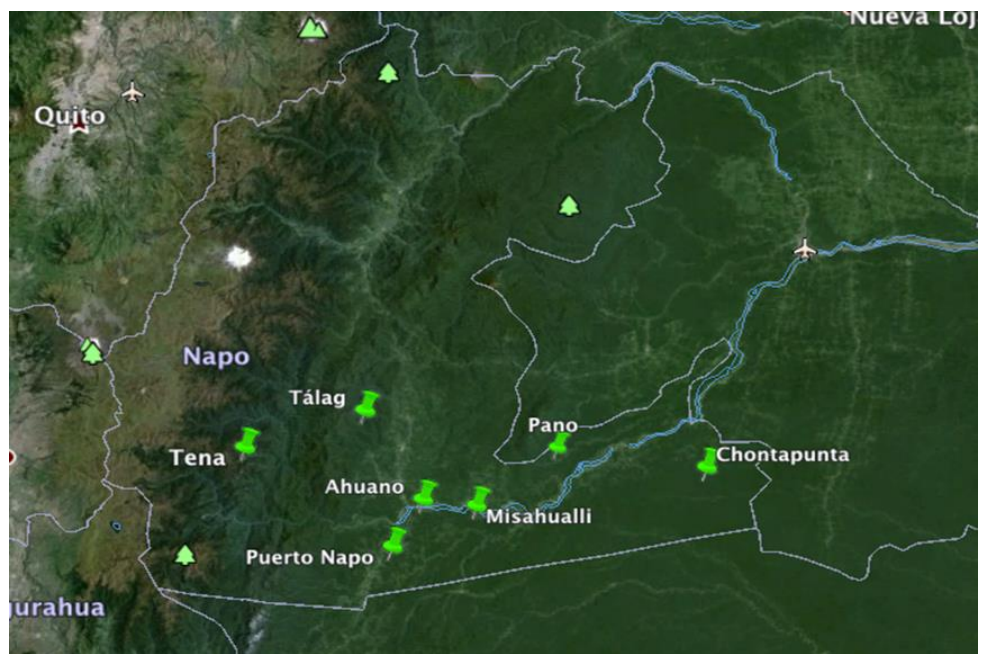

Figura 1. Parroquias de la Provincia de Napo abordadas en el presente estudio. Fuente: adaptado a partir de Google Earth, 2014.

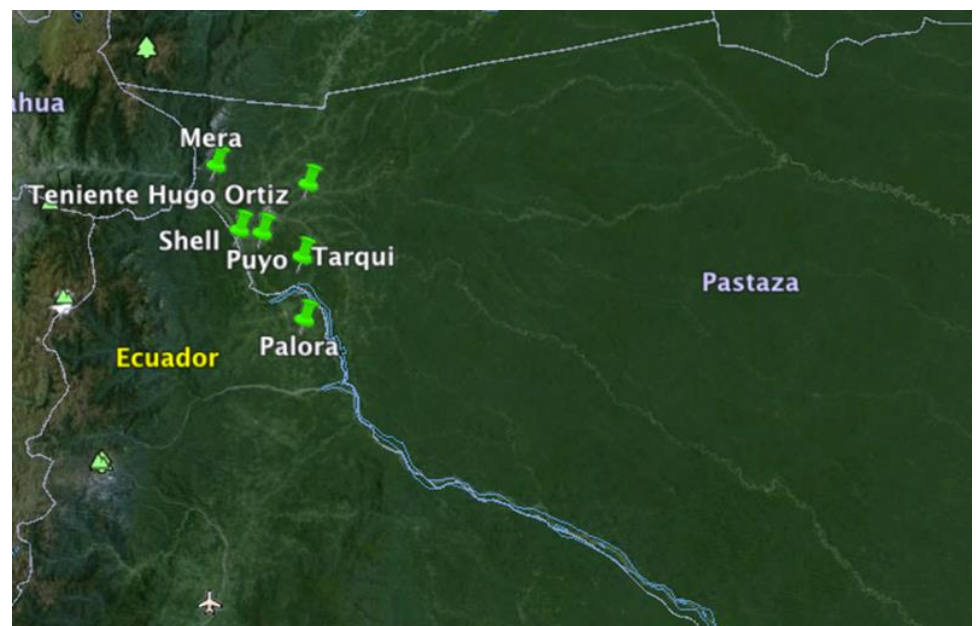

Figura 2. Parroquias de las Provincias de Pastaza y Morona Santiago abordadas en el presente estudio. Fuente: adaptado a partir de Google Earth, 2014.

En la Tabla 1 se observa el número de predios evaluados y su pertenencia a las tres provincias y a la iniciativa Socio Bosque.

Tabla No. 1. Distribución de predios del estudio en cuanto a su provincia y a la pertenencia o no a Socio Bosque

\begin{tabular}{llll}
\hline Provincia & Predios Socio Bosque & Predios No Socio Bosque & Total \\
\hline Napo & 32 & 39 & 71 \\
Pastaza & 25 & 25 & 50 \\
\hline
\end{tabular}




\begin{tabular}{llll}
\hline $\begin{array}{lll}\text { Morona Santiago } \\
\text { Total }\end{array}$ & 1 & 0 & 1 \\
& 58 & 64 & 122 \\
\hline
\end{tabular}

\section{Aplicación y tabulación de las encuestas}

Las encuestas fueron aplicadas mediante entrevistas individuales semiformales con los propietarios, acoplándose los investigadores a los horarios y disponibilidad de cada entrevistado.

Los grupos de preguntas aplicadas a través de la encuesta, se organizaron en una base de datos según su tipología: para las de la primera fase en información básica de la familia, formas de sustento, plantas que utilizan en la zona, entre otras. Para las de la segunda en seis categorías de uso de plantas: comestibles, combustibles, para madera, sociales o rituales, tóxicas y/o venenosas, medicinales (Clavijo, 2016).

\section{Análisis de la información}

Se realizaron análisis semicuantitativos con algunas preguntas selectas de las encuestas, poniendo énfasis en aquellas relacionadas con las características de uso del recurso vegetal local.

Cabe mencionar que tres investigaciones relacionadas con la presente (Granda, 2015; Clavijo, 2016; Yánez \& Granda, 2016) muestran y analizan más ampliamente todas las variables sociales y socio-ambientales registradas en la zona de estudio, en la presente nos concentramos en evaluar los aspectos relacionados con el uso cotidiano de la flora.

\section{Resultados y Discusión}

\section{Tendencias en el uso del recurso vegetal}

Esta información fue recabada en 66 de las 122 fincas, seleccionadas de entre éstas al azar, en la Fase 2 del estudio.

En la Tabla 2 se puede observar la lista de plantas reportadas como más utilizadas por las familias locales, considerando una frecuencia de uso igual o superior a cinco días al mes en al menos la tercera parte de las fincas abordadas (22 o más de las 66).

Tabla No. 2. Lista de las 68 plantas nativas e introducidas más utilizadas en las doce parroquias de estudio, considerando una frecuencia de uso igual o superior a cinco días al mes en al menos la tercera parte de las fincas abordadas.

\begin{tabular}{cllll}
\hline No. & $\begin{array}{c}\text { Nombre } \\
\text { Común }\end{array}$ & Familia & Nombre Científico & $\begin{array}{c}\text { Origen Nativo O Introducido Con } \\
\text { Respecto Al Area De Estudio }\end{array}$ \\
\hline $\mathbf{1}$ & Aguacate & Lauraceae & Persea americana & Introducida \\
$\mathbf{2}$ & Ajo de monte & Bignoniaceae & Mansoa alliacea & Nativa \\
$\mathbf{3}$ & Arroz & Poaceae & Oryza sativa & Introducida \\
$\mathbf{4}$ & Ayahuasca & Malpighiaceae & $\begin{array}{c}\text { Banisteriopsis } \\
\text { caapi }\end{array}$ & Nativa \\
\hline
\end{tabular}




\begin{tabular}{|c|c|c|c|c|}
\hline 5 & Caña de azúcar & Poaceae & $\begin{array}{l}\text { Saccharum } \\
\text { officinarum }\end{array}$ & Introducida \\
\hline 6 & Balsa & Malvaceae & $\begin{array}{l}\text { Ochroma } \\
\text { pyramidale }\end{array}$ & Nativa \\
\hline 7 & Bálsamo & Fabaceae & $\begin{array}{l}\text { Myroxylon } \\
\text { balsamum }\end{array}$ & Nativa \\
\hline 8 & Barbasco & Fabaceae & Lonchocarpus utilis & Nativa \\
\hline 9 & Cacao & Malvaceae & Theobroma cacao & Nativa \\
\hline 10 & Café & Rubiaceae & Coffea arabica & Introducida \\
\hline 11 & Caimito & Sapotaceae & Pouteria caimito & Nativa \\
\hline 12 & Camote & Convolvulaceae & Ipomoea batatas & Nativa \\
\hline 13 & Canelo & Lauraceae & Ocotea quixos & Nativa \\
\hline 14 & Caoba & Meliaceae & $\begin{array}{l}\text { Swietenia } \\
\text { macrophylla }\end{array}$ & Introducida \\
\hline 15 & Cascarilla & Rubiaceae & Cinchona officinalis & Nativa \\
\hline 16 & Cedro & Meliaceae & Cedrela odorata & Nativa \\
\hline 17 & Chiricaspi & Solanaceae & $\begin{array}{l}\text { Brunfelsia } \\
\text { grandiflora }\end{array}$ & Nativa \\
\hline 18 & Chonta & Arecaceae & Bactris gassipaes & Nativa \\
\hline 19 & Chucchuhuasu & Celastraceae & Maytenus krukovii & Nativa \\
\hline 20 & Chuncho & Fabaceae & $\begin{array}{l}\text { Cedrelinga } \\
\text { cateniformis }\end{array}$ & Nativa \\
\hline 21 & Churuyuyo & Commelinaceae & Commelina diffusa & Nativa \\
\hline 22 & Fréjol & Fabaceae & Phaseolus vulgaris & Introducida \\
\hline 23 & Guaba & Fabaceae & Inga edulis & Nativa \\
\hline 24 & Guanto & Solanaceae & $\begin{array}{l}\text { Brugmansia } \\
\text { sanguinea }\end{array}$ & Nativa \\
\hline 25 & Guarumo & Urticaceae & Cecropia peltata & Nativa \\
\hline 26 & Guayaba & Myrtaceae & Psidium guajava & Nativa \\
\hline 27 & Guayabilla & Myrtaceae & Eugenia victoriana & Nativa \\
\hline 28 & Guayacán & Bignoniaceae & $\begin{array}{l}\text { Tabebuia } \\
\text { chrysantha }\end{array}$ & Introducida \\
\hline 29 & Guayusa & Aquifoliaceae & Ilex guayusa & Nativa \\
\hline 30 & Hierba Luisa & Poaceae & $\begin{array}{l}\text { Cymbopogon } \\
\text { citratus }\end{array}$ & Introducida \\
\hline 31 & Higuerilla & Euphorbiaceae & Ricinus communis & Introducida \\
\hline 32 & Higuerón & Moraceae & Ficus insipida & Nativa \\
\hline 33 & Jengibre & Zingiberaceae & Zingiber officinale & Nativa \\
\hline 34 & Laurel & Boraginaceae & Cordia alliodora & Nativa \\
\hline 35 & Limón & Rutaceae & Citrus limon & Introducida \\
\hline 36 & Llantén & Plantaginaceae & Plantago major & Introducida \\
\hline 37 & Maíz & Poaceae & Zea mays & Nativa \\
\hline 38 & Manzanilla & Asteraceae & $\begin{array}{l}\text { Matricaria } \\
\text { chamomilla }\end{array}$ & Introducida \\
\hline
\end{tabular}




\begin{tabular}{|c|c|c|c|c|}
\hline 39 & Marco & Asteraceae & $\begin{array}{c}\text { Ambrosia } \\
\text { arborescens }\end{array}$ & Introducida \\
\hline 40 & Menta & Lamiaceae & $\begin{array}{l}\text { Mentha } \times \\
\text { rotundifolia }\end{array}$ & Introducida \\
\hline 41 & Morete & Arecaceae & Mauritia flexиosa & Nativa \\
\hline 42 & Naranja & Rutaceae & Citrus sinensis & Introducida \\
\hline 43 & Naranjilla & Solanaceae & Solanum quitoense & Nativa \\
\hline 44 & Noni & Rubiaceae & Morinda citrifolia & Introducida \\
\hline 45 & Ortiga & Urticaceae & Urtica dioica & Nativa \\
\hline 46 & Paico & Amaranthaceae & $\begin{array}{l}\text { Chenopodium } \\
\text { ambrosioides }\end{array}$ & Nativa \\
\hline 47 & Palo de Gallina & Myristicaceae & Otoba parvifolia & Nativa \\
\hline 48 & Papa & Solanaceae & Solanum tuberosum & Introducida \\
\hline 49 & Papa China & Araceae & Colocasia esculenta & Nativa \\
\hline 50 & Papaya & Caricaceae & Carica papaya & Nativa \\
\hline 51 & Pigüe & Asteraceae & Pollalesta discolor & Nativa \\
\hline 52 & Plátano seda & Musaceae & Musa acuminata & Introducida \\
\hline 53 & Plátano verde & Musaceae & Musa x paradisiaca & Introducida \\
\hline 54 & Sábila & Xanthorrhoeaceae & Aloe vera & Introducida \\
\hline 55 & $\begin{array}{l}\text { Sangre de } \\
\text { drago }\end{array}$ & Euphorbiaceae & Croton lechleri & Nativa \\
\hline 56 & Tagua & Arecaceae & $\begin{array}{l}\text { Phytelephas } \\
\text { aequatorialis }\end{array}$ & Nativa \\
\hline 57 & Tamburo & Vochysiaseae & $\begin{array}{c}\text { Vochysia } \\
\text { braceliniae }\end{array}$ & Nativa \\
\hline 58 & Tamiya muyu & Violaceae & Leonia crassa & Nativa \\
\hline 59 & Teatina & Plantaginaceae & Scoparia dulcis & Nativa \\
\hline 60 & Teta de vaca & Solanaceae & $\begin{array}{l}\text { Solanum } \\
\text { mammosum }\end{array}$ & Nativa \\
\hline 61 & Tilo & Malvaceae & Tilia officinarum & Introducida \\
\hline 62 & Toronjíl & Lamiaceae & Melissa officinalis & Introducida \\
\hline 63 & Uña de gato & Rubiaceae & Uncaria tomentosa & Nativa \\
\hline 64 & Uva de Monte & Urticaceae & $\begin{array}{c}\text { Pourouma } \\
\text { cecropiifolia }\end{array}$ & Nativa \\
\hline 65 & Valeriana & Valerianaceae & $\begin{array}{l}\text { Valeriana } \\
\text { officinalis }\end{array}$ & Introducida \\
\hline 66 & Verbena & Verbenaceae & Verbena officinalis & Introducida \\
\hline 67 & Yuca & Euphorbiaceae & Manihot esculenta & Nativa \\
\hline 68 & Guayusa & Aquifoliaceae & Ilex guayusa & Nativa \\
\hline
\end{tabular}

\section{Patrones generales de uso del recurso vegetal}

En la Tabla 3 se puede observar el número de especies vegetales que se utilizan en relación a cada categoría de uso, lo cual a su vez permite realizar una comparación cualitativa 
entre las fincas adscritas al Programa Socio Bosque (SB) y aquellas no adscritas (NSB) y el número de especies de plantas que utilizan.

El número de especies de plantas nativas utilizadas es similar en las fincas SB (38) y las NSB (42), al igual que el uso de especies introducidas en fincas SB (26) y NSB (26).

Pero al comparar el uso de plantas nativas y de introducidas, en general existe una tendencia a usar un mayor número de las primeras: en las fincas SB esta relación es de 38 a 26, y en las Fincas NSB 42 a 26.

Tabla No. 3. Número de especies utilizadas por categoría de uso registradas en el presente estudio

\begin{tabular}{lllllll}
\hline & Fincas SB & \multicolumn{2}{l}{ Fincas NSB } & \multicolumn{2}{c}{ TOTAL } \\
& Nativas & Introdu. & Nativas & Introdu. & Nativas & Introdu. \\
\hline Plantas comestibles & 8 & 10 & 10 & 10 & 13 & 10 \\
Plantas combustibles & 9 & 2 & 8 & 2 & 14 & 2 \\
Plantas para madera & 4 & 3 & 5 & 3 & 9 & 3 \\
Plantas sociales/rituales & 7 & 1 & 8 & 1 & 8 & 1 \\
Plantas venosas/tóxicas & 1 & 0 & 1 & 0 & 3 & 1 \\
Plantas medicinales & 9 & 10 & 10 & 10 & 13 & 11 \\
TOTAL & $\mathbf{3 8}$ & $\mathbf{2 6}$ & $\mathbf{4 2}$ & $\mathbf{2 6}$ & & \\
\hline
\end{tabular}

Esta tendencia de uso mayor de las plantas nativas sobre las introducidas ya ha sido reportada también en estudios en áreas similares: Alarcón (1984); Bennet (1990), Cerón (1990); Iglesias (1989).

\section{Tendencias en el uso de plantas comestibles}

Se pudo evidenciar como en el uso alimenticio predominan pocas especies de alto consumo y de origen mayormente introducido para la zona como el arroz (Oryza sativa), la caña de azúcar (Saccharum officinarum) y el plátano verde (Musa x paradisiaca); estas tres especies según de la Torre et al. (2008) son a la vez de las más consumidas en el país. Otras especies introducidas frecuentemente consumidas son la naranja (Citrus sinensis) y la papa (Solanum tuberosum).

Por otra parte, entre las especies nativas más utilizadas como alimentos destacan, en fincas SB y NSB, la chonta (Bactris gassipaes), la yuca (Manihot esculenta), la papa china (Colocasia sculenta), el morete (Mauritia flexuosa), el maíz (Zea mays), la naranjilla (Solanum quitoense) y la papaya (Carica papaya).

\section{Tendencias en el uso de plantas combustibles}

Existe poca variedad de especies utilizadas como fuente de leña, principalmente son de origen nativo, resaltando entre ellas el pigüe (Pollalesta discolor), el guarumo (Cecropia peltata y Cecropia spp.), el laurel (Cordia alliodora) y la guaba (Inga edulis), tanto en fincas SB como NSB. 
Esto puede deberse, según lo comentan Cerón y Montalvo (1998), a que ya la mayoría de hogares en la zona utilizan actualmente gas licuado de petróleo en sus cocinas, lo que hace que cada vez usen menos especies vegetales como fuente de combustibles.

Otra razón para el poco uso de plantas combustibles, según Rosero (2013), es que al estar algunas fincas adscritas al programa Socio Bosque no usan los recursos forestales para leña buscando permitir la recuperación y/o conservación del bosque.

\section{Tendencias en el uso de plantas para madera}

Para este uso, los habitantes locales prefieren una variedad relativamente reducida de especies nativas, principalmente el chuncho (Cedrelinga cateniformis), el tamburo (Vochysia sp.), el laurel (Cordia alliodora), el cedro (Cedrela odorata), la chonta (Bactris gassipaes), el canelo (Ocotea quixos), la caoba (Swietinia macrophylla), el cedro (Cedrela odorata) y el pigüe (Pollalesta discolor).

Cabe recalcar que en otros estudios, como el de Ríos et al. (2007), también se reportan estas especies como las más utilizadas para aprovechamiento y creación de productos de madera en zonas similares.

\section{Tendencias en el uso de plantas sociales o rituales}

En el análisis realizado para esta categoría, observamos que el café (Coffea arabica, especie introducida) se utiliza frecuentemente como bebida, y el cacao (Theobroma cacao), especie nativa, en forma de chocolate. Ambas son las que más se consumen por las familias estudiadas, estén o no adscritas al programa Socio Bosque. Este hábito de consumo también ha sido reportado en trabajos como los de Innerhofer y Bernhardt (2011).

Mientras que otras tres especies nativas: la guayusa (Ilex guayusa) en forma de té, y la chonta (Bactris gassipaes) y la yuca (Manihot esculenta) para preparación de chichas se consumen menos frecuentemente en esta región amazónica. Patrones similares han sido reportados por Bennet (1990) y Alarcón (1984).

\section{Tendencias en el uso de plantas venenosas o tóxicas}

Se detectaron tres especies nativas de naturaleza tóxica utilizadas eventualmente por los habitantes locales, principalmente para la pesca en ríos medianos y pequeños, de entre ellas destaca el barbasco (Lonchocarpus utilis).

\section{Tendencias en el uso de plantas medicinales}

Las plantas dentro de esta categoría son utilizadas para diferentes funciones, pero principalmente para la disminución de síntomas de diferentes enfermedades o dolencias físicas:

Para el dolor de estómago usan dos especies introducidas: la hierba luisa (Cymbopogon citratus) y la manzanilla (Matricaria chamomilla); para detener la diarrea, la nativa jengibre 
(Zingiber officinale); para disminuir la fiebre, la verbena (Verbena officinalis, introducida) o el churuyuyo (Commelina diffusa, nativa); para las heridas o golpes usan tres especies nativas: sangre de drago (Croton lechleri), chucchuhuasu (Maytenus krukovii) y el bálsamo (Myroxylon balsamum).

Finalmente, cabe también destacar al paico para tratamiento de infecciones (Chenopodium ambrosioides, nativa) y la cascarilla (Cinchona officinalis, nativa) para la malaria; para tratar hinchazones, las introducidas llantén (Plantago major) y valeriana (Valeriana officinalis); así como las nativas ajo de monte (Mansoa alliacea) para tratar tumores y la uña de gato (Uncaria tomentosa) para curar la diabetes.

La mayoría de estos usos han sido también reportados para zonas similares por otros investigadores como Marles et al. (1988), Iglesias (1989), Cerón (1990), de la Torre et al. (2008), Bennet (1990). Cabe mencionar que los informantes, las personas entrevistadas en el estudio, posiblemente no conciben los cuadros patológicos como se entienden en la medicina occidental; por lo tanto, la información etnomédica que se presenta en esta investigación debe ser tomada con la respectiva precaución.

\section{Conclusiones}

Las fincas estudiadas en las doce parroquias amazónicas tienen un nivel similar en cuanto al uso de especies de plantas nativas entre las fincas Socio Bosque y las No Socio Bosque (Tabla 3); al comparar, en cambio, el número de especies usadas entre las nativas y las introducidas, las primeras muestran un número de especies generalmente mayor al de las segundas (Tabla 3); sin embargo, en cuanto a la frecuencia de uso, las plantas introducidas se usan con mayor frecuencia con respecto a las nativas.

En cuanto a las plantas comestibles, el estudio muestra que a pesar de que existe un amplio espectro de especies nativas eventualmente utilizadas, éstas se consumen menos frecuentemente que las especies introducidas, tales como el arroz (Oryza sativa), el azúcar (Saccharum officinarum) y el plátano verde (Musa x paradisiaca).

En relación a las plantas combustibles, se observó que el uso de especies nativas para combustible (leña) es todavía relevante, aunque la variedad que se usa es baja, comparado con reportes de otros estudios en los que se utiliza mayor variedad de especies dentro de esta categoría (Bennet, 1990; Cerón, 1990; Cerón y Montalvo, 1998).

Con respecto a las plantas para madera, las especies reportadas como más utilizadas fueron el chuncho (Cedrelinga cateniformis), el tamburo (Vochysia sp.), el laurel (Cordia alliodora) y el cedro (Cedrela odorata), todas nativas de la zona; estas especies han sido reportadas para uso maderable también por Mejía y Pacheco (2013). Se observó que, al igual que con la leña, se usa dentro de esta categoría poca variedad de especies.

En cuanto a plantas sociales o rituales, las especies más utilizadas fueron el café (Coffea arabica), el cacao (Theobroma cacao), la chonta (Bactris gassipaes), la yuca (Manihot esculenta) y la guayusa (Ilex guayusa): una especie introducida y cuatro nativas. 
Dentro de las plantas tóxicas y/o venenosas, la más utilizada es el barbasco (Lonchocarpus utilis).

Finalmente las veinte y cuatro especies medicinales evaluadas fueron reportadas como usadas en la mayoría de fincas pero con frecuencias de uso bajas.

Se puede mencionar qué factores como la migración, el desconocimiento, la expansión de la frontera agrícola, las actividades petroleras y los procesos de transculturación, han sido influyentes en la disminución del conocimiento y nivel de uso de la flora nativa de la zona; lo cual también ha sido afirmado en estudios como los de Ríos et al. (2007).

\section{Recomendaciones}

Es aconsejable realizar estudios similares en otros sectores y provincias de la región amazónica ecuatoriana para obtener más información sobre la dinámica del uso de las especies vegetales y así poder proponer planes de manejo de las especies (tales como los recomendados por Yánez, 1993; Yánez, 2006) y/o planes de recuperación del conocimiento ancestral del uso de plantas nativas de esta región, así como su conservación futura, ya que todo este conocimiento es parte de nuestra cultura e idiosincrasia.

Las investigaciones deberán realizarse con la debida planificación y el respeto a las comunidades campesinas e indígenas que fueren real o potencialmente abordadas, buscando levantar información sobre el conocimiento de uso, pero también sobre las posibles formas de aplicación futura de estos recursos naturales en bien de la sociedad ecuatoriana.

Es recomendable organizar talleres de concientización sobre el conocimiento y uso de la flora nativa para la gente local, fomentando el incremento de este recurso, especialmente en el caso de ser fuente alimenticia, y así no sea desplazado por especies introducidas que provienen de agroecosistemas tropicales, que como lo menciona Yánez (2013), en los que predominan los monocultivos.

Asimismo, se deben realizar estudios sobre especies nativas de fácil regeneración que puedan ser sustitutos de combustibles fósiles en zonas rurales locales, para ello se necesita más información que permita desarrollar planes de aprovechamiento sostenible de algunas plantas nativas como fuente de combustible (leña).

Iniciar un estudio de las formas de manejo de árboles nativos que mantienen los dueños de predios para la producción y venta de madera, esto permitirá formular un plan de manejo de aprovechamiento de este recurso que se adapte a las condiciones que tenemos en esta zona, coadyuvando a la conservación del ecosistema.

Se debe levantar más información de plantas de importancia social y ritual para recuperar saberes ancestrales en sectores de mayor diversidad vegetal de la Amazonía Ecuatoriana, esto facilitará tener un banco de información ancestral que permita sentar una base para estudios y aplicaciones futuras como las ejemplificadas por Yánez et al. (2015). 
Al igual que con las plantas sociales y rituales se recomienda levantar mayor información sobre las plantas venenosas o tóxicas nativas y su uso en relación con el desarrollo de prácticas ancestrales de caza y pesca en las comunidades amazónicas.

En cuanto a las plantas medicinales se recomienda que se conforme un banco de información sobre los usos y conocimiento ancestrales relacionados con ellas que poseen los habitantes de zonas amazónicas, que permita el desarrollo de estudios científicos más profundos y el eventual desarrollo de aplicaciones futuras en medicina.

\section{Bibliografía}

Alarcón, R. (1984). Etnobotánica de los Quichuas de la Amazonía Ecuatoriana. Tesis de Licenciatura en Ciencias Biológicas. Facultad de Ciencias de la Educación. Quito. Pontificia Universidad Católica del Ecuador, 159p.

Bennet, B. (1990). Useful plants of Amazonia Ecuador. New York. U.S. Agency For International Development.

Bonete, M., Urquizo, C., Guevara, R., \& Yánez, P. (2016). Estudio de cuatro tubérculos y raíces tuberosas no tradicionales de la sierra centro de Ecuador y su potencial de uso en platos de autor. Qualitas, 12, 37-67.

Cerón, C. (1990). Manejo florístico Shuar-Achuar (Jibaro) del Ecosistema Amazónico del Ecuador. Ecuador. Ciencia y Tecnología, 9p.

Cerón, C. y C. Montalvo. (1998). Etnobotánica de los Huaorani de Quehueiri-ono, Napo, Ecuador. FUNDACYT, Ediciones Abya-Yala y Herbario Alfredo Paredes (QAP), Escuela de Biología, Universidad Central del Ecuador. Quito, 232pp.

Clavijo Páez, J. C. (2016). Patrones de uso de la flora y su relación con actividades de conservación de bosques nativos en doce parroquias amazónicas de Ecuador. Trabajo de Titulación en Biología Ambiental, Universidad Internacional del Ecuador, Quito.

Cunningham, A. (2001). Etnobotánica aplicada: pueblos, uso de plantas silvestres y conservación. Manual de la Serie Pueblos y Plantas. Uruguay, Editorial Nordan-Comunidad, Vol. 4, 310 .

De la Torre, L., H. Navarrete, P. Muriel M., M. J. Macía y H. Balslev. (2008). Enciclopedia de las Plantas Útiles del Ecuador. Quito. Herbario QCA y Herbario AAU, 942 p.

Google Earth. (2014). Imágenes de la Región Amazónica Ecuatoriana. Revisadas entre mayo a agosto de 2014.

Granda Muñoz, M. J. (2015). Análisis Socio-Ambiental en doce parroquias amazónicas de Ecuador y su relación con actividades de conservación de bosques nativos. Trabajo de Titulación en Biología Ambiental, Universidad Internacional del Ecuador, Quito. 
Iglesias, G. (1989). El uso de las plantas en la medicina tradicional de los Quichuas del Napo. Quito, Abya Yala, Sacha Jambi.

Innerhofer, S., \& Bernhardt, C. (2011). Ethnobotanic garden design in the Ecuadorian Amazon. Biodivers Conserv 20:429-439.

Marles, R., Neill, D., \& Farnsworth, N. (1988). A contribution to the Ethnopharmacology of the lowland Quichua people of Amazonian Ecuador. Pharmaceutical Sciences. College of Pharmacy, University of Illinois. Missouri Botanical Garden, 10p.

Mejía, E., \& Pacheco, P. (2013). Aprovechamiento forestal y mercados de la madera en la Amazonía Ecuatoriana. Occasional Paper 97. Bogor, Indonesia: CIFOR, 99p.

Palacios, W., \& Malessa, U. (2010). Situación de las comunidades productoras forestales de la Amazonía ecuatoriana: obstáculos y oportunidades para comercializar madera legal. Quito. TRAFFIC, Oficina regional de América del Sur, 16p.

Ríos, M., Koziol, M., Pedersen, H. \& Granda, G. (2007). Plantas Útiles del Ecuador: Aplicaciones, retos y perspectivas. Quito. Ediciones Abya-Yala, 652p.

Rosero, C. (2013). Sistematización del Programa Nacional de Incentivos Socio Bosque. Ministerio del Ambiente. Quito, 66p.

Uquillas, J. (1981). Colonización y asentamientos espontáneos en la Amazonia ecuatoriana. Quito. Instituto Nacional de Colonización de la Región Amazónica Ecuatoriana, 31p.

Yánez, A. P. (1993). Estructura Poblacional, Fenología Reproductiva y Evaluación Económica de tres especies del género Pourouma (Cecropiaceae) en la Región del Alto Río Napo, Ecuador. Tesis de Licenciatura en Biología, PUCE-Q, Quito.

Yánez, P. (1999). Distribución geográfica y aspectos etnobotánicos de tres especies del género Pourouma ("uva de monte”), Cecropiaceae, en la región amazónica de Ecuador. Rev. Forest. Venez, 43(1), 103-109.

Yánez, P. (2006). Plan de uso y manejo de la guaviduca (Piper carpunya Ruiz \& Pav., Piperaceae) para la comunidad de Chiriboga y áreas adyacentes, Pichincha-Ecuador. Programa de Manejo de la Biodiversidad y Biocomercio (PMBB)-EcoCiencia. Informe final presentado al Programa de Facilitación del Biocomercio/UNCTAD. Quito.

Yánez, P. (2012). Consideraciones para el diseño y aplicación de planes de manejo de especies vegetales silvestres no maderables de interés comercial. Qualitas, 4, 31-40.

Yánez, P. (2013). La pérdida de los bosques tropicales: algunos de sus efectos sobre la estabilidad de nuestro Planeta. Qualitas, 6, 74-78. 
Yánez, P. (2014). Ecología y biodiversidad: un enfoque desde el neotrópico. Quito: UNIBE/UIDE. $172 \mathrm{pp}$.

Yánez, P., Rivadeneira, L., Balseca, D., \& Larenas, C. (2015). Características morfológicas y de concentración de capsaicina en cinco especies nativas del género Capsicum cultivadas en Ecuador. La Granja: Revista de Ciencias de la Vida, 22(2), 12-32.

Yánez, P., \& Granda, M. J. (2016). Factores socio-ambientales y de conservación en predios amazónicos de Ecuador vinculados o no al Programa Socio Bosque. INNOVA Research Journal, 1(11), 17-29. 